\title{
Stagnation-Point Flow and Heat Transfer over a Nonlinearly Stretching/Shrinking Sheet in a Micropolar Fluid
}

\author{
Khairy Zaimi ${ }^{1}$ and Anuar Ishak ${ }^{2}$ \\ ${ }^{1}$ Institute of Engineering Mathematics, Universiti Malaysia Perlis, Pauh Putra Campus, 02600 Arau, Perlis, Malaysia \\ ${ }^{2}$ School of Mathematical Sciences, Faculty of Science and Technology, Universiti Kebangsaan Malaysia (UKM), \\ 43600 Bangi, Selangor, Malaysia \\ Correspondence should be addressed to Anuar Ishak; anuarishak@yahoo.com
}

Received 3 December 2013; Revised 5 May 2014; Accepted 6 May 2014; Published 21 May 2014

Academic Editor: Changbum Chun

Copyright (C) $2014 \mathrm{~K}$. Zaimi and A. Ishak. This is an open access article distributed under the Creative Commons Attribution License, which permits unrestricted use, distribution, and reproduction in any medium, provided the original work is properly cited.

\begin{abstract}
This paper considers the problem of a steady two-dimensional stagnation-point flow and heat transfer of an incompressible micropolar fluid over a nonlinearly stretching/shrinking sheet. A similarity transformation is employed to convert the partial differential equations into nonlinear ordinary ones which are then solved numerically using a shooting method. Numerical results obtained are presented graphically, showing the effects of the micropolar or material parameter and the stretching/shrinking parameter on the flow field and heat transfer characteristics. The dual solutions are found to exist in a limited range of the stretching/shrinking parameter for the shrinking case, while unique solutions are possible for all positive values of the stretching/shrinking parameter (stretching case). It is also observed that the skin friction coefficient and the magnitude of the local Nusselt number increase as the material parameter increases.
\end{abstract}

\section{Introduction}

The number of investigations in the convective flow and heat transfer over a stretching or shrinking sheet has grown dramatically in recent years. The development of research in this area is stimulated by the presence of a variety of its real world applications in industrial and engineering processes. Extrusion, glass fiber, glass blowing, paper production, and extraction of polymer and rubber sheets are examples of these applications. Crane [1] was the first to report the analytical solution for the boundary layer flow of an incompressible viscous fluid over a stretching plate. On the other hand, it seems that Miklavčič and Wang [2] were the first who investigated the flow over a shrinking sheet. As reported by Miklavčič and Wang [2], the boundary layer flow over a shrinking sheet is likely to exist in two conditions, firstly, by imposing an adequate suction on the boundary [2] and, secondly, by considering a stagnation flow [3]. After the pioneering contributions by both Crane [1] and Miklavčič and Wang [2], the study of fluid flow over a stretching/shrinking sheet has been explored by a large number of researchers under different physical conditions.

In recent years, the investigations of flow in the stagnation region over a stretching/shrinking surface in a micropolar fluid have been the subject of interest among researchers. The pioneering work on this fluid was given by Eringen [4] when introducing the first theory on micropolar fluids. This theory considers the microstructure and micromotions of the fluid which were unable to be explained by the classical theory of Newtonian fluid. This theory has attracted many researchers to proceed on further research, because it was seen to be capable of explaining the behavior of more complex fluids such as lubricants, paints, polymers, and animal blood, in which the classical Newtonian fluids theory is inadequate. In order to apply the theory of micropolar fluids, a transport equation representing the principle of conservation of local angular momentum to the usual transport equations for the conservation of mass and momentum must be added [5]. As a result, one additional local constitutive parameter has been introduced. A good list of published papers 
investigating several aspects of flow in a micropolar fluid has been presented by Nazar et al. [5], Lok et al. [6], Ishak et al. [7, 8], Ziabakhsh et al. [9], Attia [10], Hayat et al. [11, 12], Yacob et al. [13], Das [14], and Zheng et al. [15] among others. However, we notice that the study of fluid flow over a nonlinearly stretching/shrinking sheet in a micropolar fluid has not received much consideration. Hayat et al. [11, 12] studied the problem of stagnation-point flow toward a nonlinearly stretching surface in a micropolar fluid. However, the solutions reported by Hayat et al. [11] are only locally similar. Ziabakhsh et al. [9] employed a transformation which reduces the momentum and angular momentum equations to a single equation. It seems that the similarity solution for the problem of fluid flow over a nonlinearly stretching sheet in a micropolar fluid is possible only if the momentum and angular momentum equations are reduced to a single equation by an appropriate transformation. In the present paper, we study the problem of two-dimensional stagnationpoint flow and heat transfer of a micropolar fluid over a nonlinearly stretching/shrinking sheet. Numerical results are obtained by solving the nonlinear similarity equations using a shooting method. The effects of the governing parameters on the flow field and heat transfer characteristics are graphically presented and discussed.

\section{Problem Formulation}

Consider a two-dimensional flow of an incompressible micropolar fluid towards a stagnation point on a nonlinearly stretching/shrinking sheet. The stretching/shrinking velocity $u_{w}(x)$, free stream velocity $u_{e}(x)$, and the temperature of the surface $T_{w}(x)$ are assumed to vary nonlinearly from the stagnation point, that is, $u_{w}(x)=b x^{n}, u_{e}(x)=a x^{n}$, and $T_{w}(x)=T_{\infty}+c x^{2 n}$, where $a, b, c$, and $n$ are constants, with $a>$ 0 and $b, c, n \geq 0$. It is also assumed that the temperature far from the surface of the sheet is $T_{\infty}$. Under these assumptions, the basic equations governing the flow are (see [9] and [11])

$$
\begin{gathered}
\frac{\partial u}{\partial x}+\frac{\partial v}{\partial y}=0 \\
u \frac{\partial u}{\partial x}+v \frac{\partial u}{\partial y}=u_{e} \frac{d u_{e}}{d x}+\frac{\mu+\kappa}{\rho} \frac{\partial^{2} u}{\partial y^{2}}+\frac{\kappa}{\rho} \frac{\partial N}{\partial y} \\
\rho j\left(u \frac{\partial N}{\partial x}+v \frac{\partial N}{\partial y}\right)=\gamma \frac{\partial^{2} N}{\partial y^{2}}-\kappa\left(2 N+\frac{\partial u}{\partial y}\right), \\
u \frac{\partial T}{\partial x}+v \frac{\partial T}{\partial y}=\frac{k}{\rho c_{p}} \frac{\partial^{2} T}{\partial y^{2}}
\end{gathered}
$$

where $u$ and $v$ are the velocity components in the $x$ and $y$ directions, respectively, $T$ is the fluid temperature, $N$ is the component of the microrotation vector normal to the $x-y$ plane, $\mu$ is the dynamic viscosity, $\kappa$ is the vortex viscosity (or the microrotation viscosity), $\rho$ is the fluid density, $j$ is the microinertia density, $k$ is the thermal conductivity, $\gamma$ is the spin gradient viscosity, and $c_{p}$ is the specific heat at constant pressure.
The boundary conditions of (1)-(4) are

$$
\begin{gathered}
u=u_{w}, \quad v=0, \quad N=-m \frac{\partial u}{\partial y}, \\
T=T_{w} \quad \text { at } y=0 \\
u \longrightarrow u_{e}, \quad N \longrightarrow 0, \quad T \longrightarrow T_{\infty} \text { as } y \longrightarrow \infty,
\end{gathered}
$$

where $m$ is a constant with $0 \leq m \leq 1$. The case $m=1 / 2$ indicates the vanishing of the antisymmetric part of the stress tensor and denotes a weak concentration of microelements (Ahmadi [17]), which will be studied here. Following the works by Ishak et al. [8], Ahmadi [17], and Yücel [18] we assume that $\gamma=(\mu+\kappa / 2) j=\mu(1+K / 2) j$, where $K=\kappa / \mu$ is the micropolar or material parameter. This assumption is required to allow the field of equations to predict the correct behavior in the limiting case when the microstructure effects become negligible and the total spin $N$ reduces to the angular velocity.

To seek the similarity solutions of (1)-(4), subject to the boundary conditions (5), we employ the following similarity transformation (see Ishak et al. [8] and Ziabakhsh et al. [9]):

$$
\begin{aligned}
\eta & =\left(\frac{u_{e}}{v x}\right)^{1 / 2} y, \quad \psi=\left(\nu x u_{e}\right)^{1 / 2} f(\eta), \\
N & =u_{e}\left(\frac{u_{e}}{v x}\right)^{1 / 2} h(\eta), \\
h(\eta) & =-\frac{1}{2} f^{\prime \prime}(\eta), \quad \theta(\eta)=\frac{T-T_{\infty}}{T_{w}-T_{\infty}},
\end{aligned}
$$

where prime denotes a differentiation with respect to $\eta$ (the similarity variable) and $\psi$ is the stream function, which is defined as $u=\partial \psi / \partial y$ and $v=-\partial \psi / \partial x$, that satisfies the continuity equation (1). The quantity $v=\mu / \rho$ is the kinematic viscosity, $f$ is the dimensionless stream function, $\theta$ is the dimensionless temperature, and $h$ is the dimensionless microrotation. Substituting (6) into (2)-(4), (2) and (3) are transformed into a single nonlinear ordinary differential equation (7) while the energy equation (4) reduces to (8) as follows:

$$
\begin{gathered}
\left(1+\frac{K}{2}\right) f^{\prime \prime \prime}+\frac{n+1}{2} f f^{\prime \prime}-n f^{\prime 2}+n=0, \\
\frac{1}{\operatorname{Pr}} \theta^{\prime \prime}+\frac{n+1}{2} f \theta^{\prime}-2 n f^{\prime} \theta=0 .
\end{gathered}
$$

The boundary conditions (5) become

$$
\begin{gathered}
f(0)=0, \quad f^{\prime}(0)=\varepsilon, \quad \theta(0)=1 \\
f^{\prime}(\eta) \longrightarrow 1, \quad \theta(\eta) \longrightarrow 0 \quad \text { as } \eta \longrightarrow \infty,
\end{gathered}
$$

where $\operatorname{Pr}$ is the Prandtl number and $\varepsilon$ is the stretching/shrinking parameter (or velocity ratio parameter) defined as

$$
\operatorname{Pr}=\frac{\mu c_{p}}{k}, \quad \varepsilon=\frac{b}{a},
$$

with $\varepsilon>0$ for stretching and $\varepsilon<0$ for shrinking. 
TABLE 1: The values of $f^{\prime \prime}(0)$ for different values of $K$ when $\operatorname{Pr}=1$ and $n=1$ for both stretching and shrinking cases.

\begin{tabular}{lcc}
\hline$K$ & $\varepsilon$ & Present \\
\hline 0 & 0.5 & 0.713294 \\
& -1.2 & $0.932473(0.233649)$ \\
0.1 & 0.5 & 0.696104 \\
& -1.2 & $0.910000(0.228018)$ \\
0.5 & 0.5 & 0.637990 \\
& -1.2 & $0.834029(0.208982)$ \\
1 & 0.5 & 0.582402 \\
& -1.2 & $0.761361(0.190771)$ \\
2 & 0.5 & 0.504375 \\
& -1.2 & $0.659359(0.165169)$ \\
5 & 0.5 & 0.381272 \\
& -1.2 & $0.498427(0.124891)$ \\
\hline
\end{tabular}

Note: ( ) second solution.

The quantities of physical interest in the present study are the skin friction coefficient $C_{f}$ and the local Nusselt number $\mathrm{Nu}_{x}$ which are defined as

$$
C_{f}=\frac{\tau_{w}}{\rho u_{e}^{2}}, \quad \mathrm{Nu}_{x}=\frac{x q_{w}}{k\left(T_{w}-T_{\infty}\right)},
$$

where $\tau_{w}$ and $q_{w}$ are the surface shear stress and the surface heat flux, respectively, which are defined as

$$
\tau_{w}=\left[(\mu+\kappa) \frac{\partial u}{\partial y}+\kappa N\right]_{y=0}, \quad q_{w}=-k\left(\frac{\partial T}{\partial y}\right)_{y=0} .
$$

Using (6), (11), and (12), we obtain

$$
C_{f} \operatorname{Re}_{x}^{1 / 2}=\left(\frac{1+K}{2}\right) f^{\prime \prime}(0), \quad \frac{\mathrm{Nu}_{x}}{\operatorname{Re}_{x}^{1 / 2}}=-\theta^{\prime}(0),
$$

where $\operatorname{Re}_{x}=u_{e} x / v$ is the local Reynolds number.

\section{Results and Discussion}

The nonlinear ordinary differential equations (7) and (8) subjected to the boundary conditions (9) were solved numerically by the shooting method with the help of the shootlib function in Maple software. This method is described in the book by Jaluria and Torrance [19] and has been applied extensively by the present authors; see Yacob et al. [13] and Wan Zaimi and Ishak [20]. The results obtained illustrate the effects of some governing parameters on the velocity and temperature profiles as well as the skin friction coefficient and the local Nusselt number (representing the heat transfer rate at the surface). Dual solutions were obtained using this method by employing different initial guesses for the unknown values of $f^{\prime \prime}(0)$ and $-\theta^{\prime}(0)$ where all velocity and temperature profiles satisfy the infinity boundary conditions (9) asymptotically but with different shapes and boundary layer thicknesses.

The values of $f^{\prime \prime}(0)$ and $-\theta^{\prime}(0)$ are shown in Tables 1 and 2, respectively, for different values of the material parameter $K$. It is seen that dual solutions of (7)-(9) exist for
TABLE 2: The values of the local Nusselt number $-\theta^{\prime}(0)$ for different values of $K$ when $\operatorname{Pr}=1$ and $n=1$ for both stretching and shrinking cases.

\begin{tabular}{lcc}
\hline$K$ & $\varepsilon$ & Present \\
\hline 0 & 0.5 & 1.313579 \\
& -1.2 & $-1.245972(31.187615)$ \\
0.1 & 0.5 & 1.310959 \\
& -1.2 & $-1.315415(18.408602)$ \\
0.5 & 0.5 & 1.301719 \\
& -1.2 & $-1.600926(7.393405)$ \\
1 & 0.5 & 1.292284 \\
& -1.2 & $-1.986747(4.472306)$ \\
2 & 0.5 & 1.277931 \\
& -1.2 & $-2.936899(2.664126)$ \\
5 & 0.5 & $-13.150690(1.350500)$ \\
\hline
\end{tabular}

Note: ( ) second solution.

TABLE 3: Values of $f^{\prime \prime}(0)$ for different values of $n$ when $m=0.5$, $\varepsilon=0$, and $K=0$.

\begin{tabular}{lccc}
\hline$n$ & Wang [3] & Ishak et al. [16] & Present \\
\hline 0.5 & & & 0.8997168 \\
1 & 1.2325876 & 1.2325876 & 1.2325876 \\
1.5 & & & 1.4933820 \\
2 & & & 1.7150679 \\
3 & & & 2.0891103 \\
\hline
\end{tabular}

the shrinking case. To validate the numerical results obtained, we compare the values of $f^{\prime \prime}(0)$ with those obtained by Wang [3] and Ishak et al. [16], as presented in Table 3, which shows good agreement.

Figures 1 and 2 illustrate the variation of the skin friction coefficient and the local Nusselt number as a function of the stretching/shrinking parameter $\varepsilon$ for some values of the micropolar parameter $K$ when $n=1$ and $\operatorname{Pr}=1$. We classify the first and second solutions based on how they appear in Figure 2; that is, the first solution has greater values of the skin friction coefficient $C_{f} \operatorname{Re}_{x}^{1 / 2}$ as compared to those of the second solutions. It is found that dual solutions exist for the shrinking case $(\varepsilon<0)$ while unique solution is obtained for the stretching case $(\varepsilon>0)$. The solution exists up to a critical value of $\varepsilon$, say $\varepsilon_{c}$. There are no solutions of (7) and (8) subjected to the boundary conditions (9) when $\varepsilon<$ $\varepsilon_{c}<0$. Based on our computations, this critical value $\varepsilon_{c}$ is -1.24657 for each value of $K=0,0.5,1$, and 2 . The stability analysis of multiple solutions has been carried out by several researchers such as Merkin [21], Weidman et al. [22], Paullet and Weidman [23], Harris et al. [24], Postelnicu and Pop [25], and most recently by Rosca and Pop [26] for similar flow situation. Their analysis has found that the first solution is physically stable and applicable in practice, whilst the second solutions are not. Thus, we postulate this finding to be valid in reference to the present results and we are not repeating the analysis here.

In Figure 1, it is clearly shown that an increase in the micropolar parameter $K$ leads to an increase in the skin 


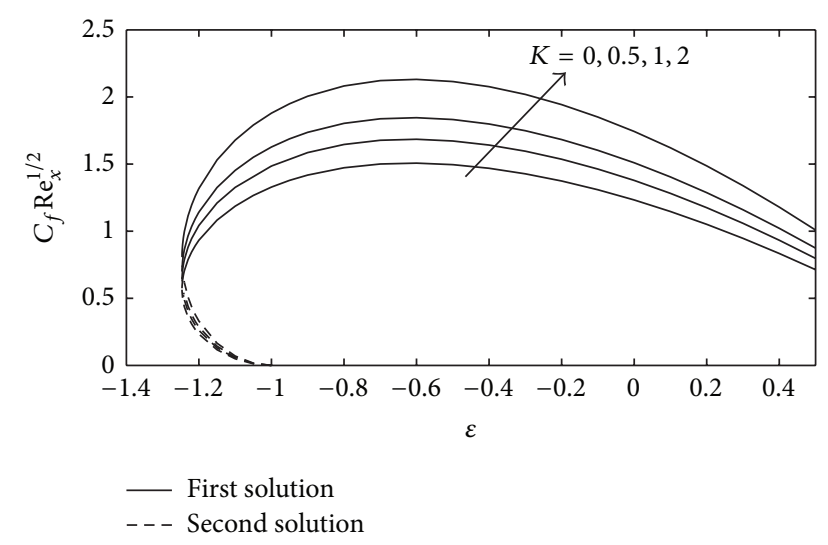

FIGURE 1: Variation of the skin friction coefficient $C_{f} \operatorname{Re}_{x}^{1 / 2}$ with $\varepsilon$ for different values of $K$ when $n=1$ and $\operatorname{Pr}=1$.

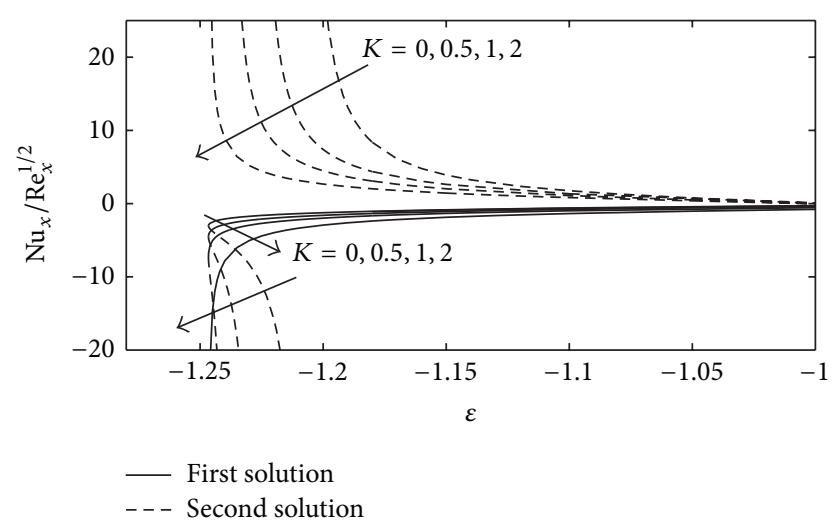

FIGURE 2: Variation of the local Nusselt number $\mathrm{Nu}_{x} / \mathrm{Re}_{x}^{1 / 2}$ with $\varepsilon$ for different values of $K$ when $n=1$ and $\operatorname{Pr}=1$.

friction coefficient for the first solution. This observation is found because of the fact that the micropolar parameter effect increases the surface shear stress and in consequence increases the velocity gradient at the surface $f^{\prime \prime}(0)$. As a result, the skin friction coefficient $C_{f} \mathrm{Re}_{x}^{1 / 2}$ increases as illustrated in Figure 1.

Figure 2 shows the variation of the local Nusselt number $\mathrm{Nu}_{x} / \operatorname{Re}_{x}^{1 / 2}$ with $\varepsilon$ when $\operatorname{Pr}=1$ and $n=1$. For the first solution, the magnitude of the local Nusselt number representing the heat transfer rate at the surface increases with increasing values of the micropolar parameter $K$ as presented in Figure 2. This finding could be due to the increasing temperature gradient at the surface as parameter $K$ increases. It is also found that unique solutions do exist for all positive values of the stretching/shrinking parameter $\varepsilon$ in the stretching region much higher than those reported in Table 2. However, the Nusselt number $-\theta^{\prime}(0)$ for the second solution becomes unbounded as $\varepsilon \rightarrow-1.20^{+},-1.22^{+},-1.23^{+}$ and as $\varepsilon \rightarrow-1.20^{-},-1.22^{-},-1.23^{-}$for each $K=0,0.5$, and 1 , respectively.

Figure 3 is depicted to examine the effect of the micropolar parameter $K$ on the velocity for the shrinking case $\varepsilon=-1.2$. It is seen that the velocity of the fluid decreases as $K$ increases for both solutions as shown in Figure 3. Furthermore, increasing $K$ is to increase the velocity boundary layer

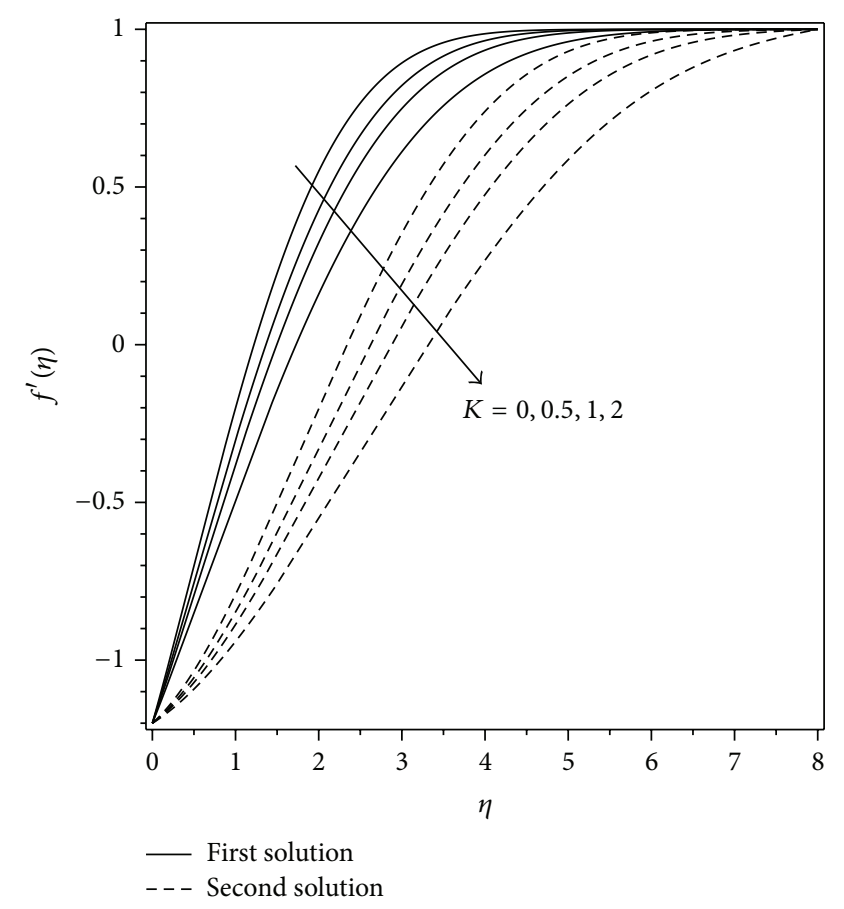

FIGURE 3: Effect of the material parameter $K$ on the velocity profiles $f^{\prime}(\eta)$ when $n=1, \operatorname{Pr}=1$, and $\varepsilon=-1.2$ (shrinking case).

thickness and that in turn decreases the velocity gradient at the surface $f^{\prime \prime}(0)$.

The influence of the micropolar parameter $K$ on the temperature profiles is displayed in Figure 4. It is evident from Figure 4 that increasing $K$ is to increase the temperature gradient at the surface (in absolute sense) for the first solution while the trend is opposite for the second solution. This result is in agreement with the results presented in Figure 2.

Figures 5 and 6 show the velocity and temperature profiles, respectively, for different values of $n$ when $K=1$ and $\operatorname{Pr}=1$ for the shrinking case $\varepsilon=-1.2$. For the first solution, which we expect to be the physically realizable solution, the velocity gradient at the surface increases as $n$ increases, but an opposite trend is observed for the temperature gradient at the surface. Thus, the local Nusselt number decreases as $n$ increases. We note that $n=0$ corresponds to uniform surface temperature.

In addition, Figures 3-6 indicate that both velocity and temperature profiles reach the far field boundary conditions (9) asymptotically, hence supporting the validity of the numerical results, besides supporting the existence of the dual solutions presented in Figures 1 and 2. From Figures 3-6, it is also clearly shown that both the velocity and thermal boundary layer thicknesses for the second solutions are larger compared to those of the first solutions. This observation supports the instability of the second solutions mentioned in the previous discussion.

\section{Conclusions}

In the present study, we investigated numerically the stagnation-point flow and heat transfer over a nonlinearly 


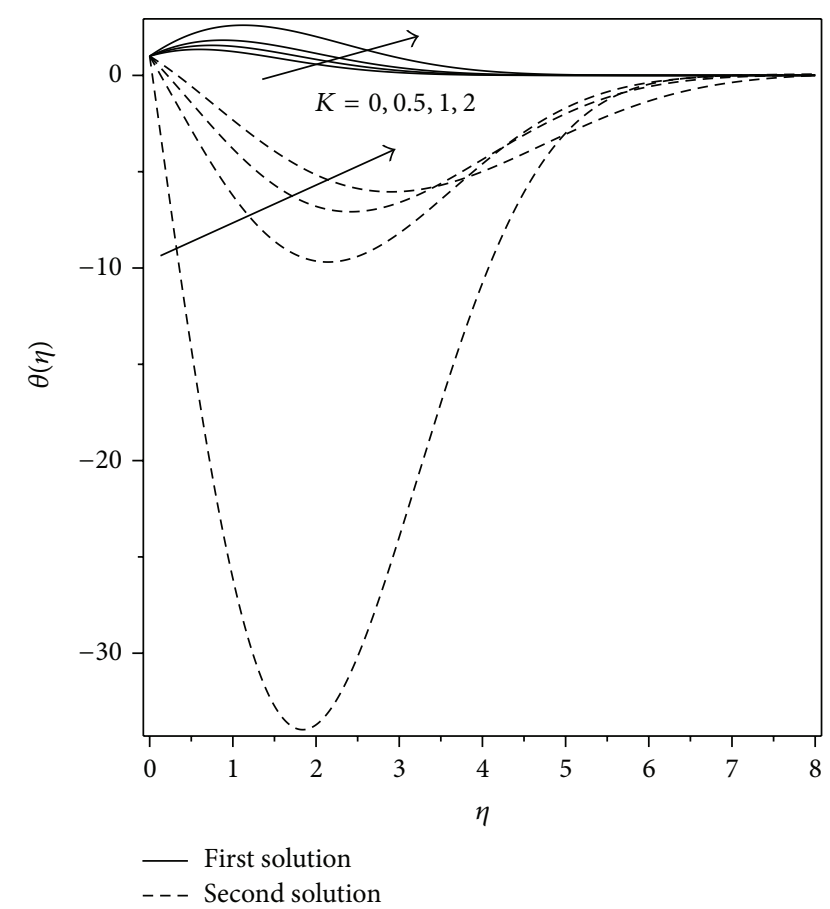

Figure 4: Effect of the material parameter $K$ on the temperature profiles $\theta(\eta)$ when $n=1, \operatorname{Pr}=1$, and $\varepsilon=-1.2$ (shrinking case).

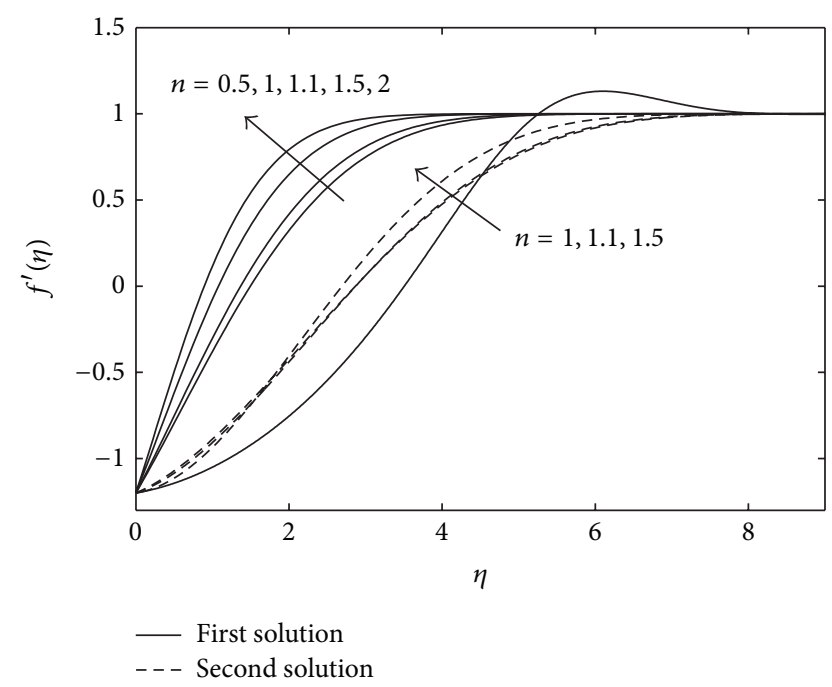

Figure 5: The velocity profiles $f^{\prime}(\eta)$ for some values of $n$ when $K=$ $1, \operatorname{Pr}=1$, and $\varepsilon=-1.2$ (shrinking case).

stretching/shrinking sheet in a micropolar fluid. The graphical representation and discussion focused on the effects of the material and stretching/shrinking parameters on the flow and the thermal fields. Dual solutions were found for a certain range of the shrinking domain, while, for the stretching domain, the solution is unique. For the first solution (stable solution), the material parameter increases both the skin friction coefficient and the magnitude of the local Nusselt number.

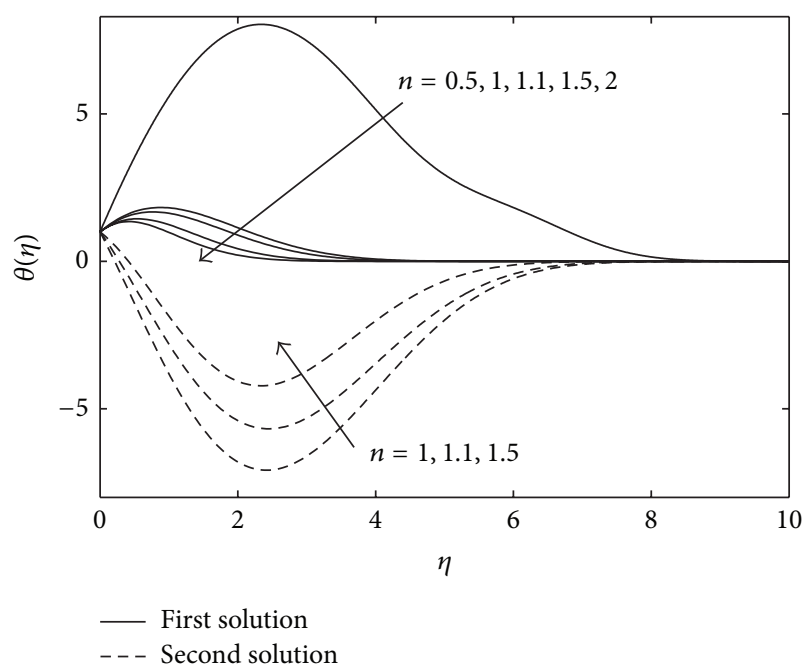

Figure 6: The temperature profiles $\theta(\eta)$ for some values of $n$ when $K=1, \operatorname{Pr}=1$, and $\varepsilon=-1.2$ (shrinking case).

\section{Conflict of Interests}

The authors declare that there is no conflict of interests regarding the publication of this paper.

\section{Acknowledgments}

The authors wish to express their thanks to the anonymous referees for their valuable comments and suggestions. The financial supports received from the Ministry of Higher Education, Malaysia (Project Code: FRGS/1/2012/ SG04/UKM/01/1) and the Universiti Kebangsaan Malaysia (Project Code: DIP-2012-31) are gratefully acknowledged.

\section{References}

[1] L. J. Crane, "Flow past a stretching plate," Zeitschrift für Angewandte Mathematik und Physik ZAMP, vol. 21, no. 4, pp. 645-647, 1970.

[2] M. Miklavčič and C. Y. Wang, "Viscous flow due to a shrinking sheet," Quarterly of Applied Mathematics, vol. 64, no. 2, pp. 283290, 2006.

[3] C. Y. Wang, "Stagnation flow towards a shrinking sheet," International Journal of Non-Linear Mechanics, vol. 43, no. 5, pp. 377382, 2008.

[4] A. C. Eringen, “Theory of thermomicrofluids," Journal of Mathematical Analysis and Applications, vol. 38, no. 2, pp. 480-496, 1972.

[5] R. Nazar, N. Amin, D. Filip, and I. Pop, "Stagnation point flow of a micropolar fluid towards a stretching sheet," International Journal of Non-Linear Mechanics, vol. 39, no. 7, pp. 1227-1235, 2004.

[6] Y. Y. Lok, I. Pop, and A. J. Chamkha, "Non-orthogonal stagnation-point flow of a micropolar fluid," International Journal of Engineering Science, vol. 45, no. 1, pp. 173-184, 2007.

[7] A. Ishak, R. Nazar, and I. Pop, "Magnetohydrodynamic (MHD) flow of a micropolar fluid towards a stagnation point on 
a vertical surface," Computers \& Mathematics with Applications, vol. 56, no. 12, pp. 3188-3194, 2008.

[8] A. Ishak, R. Nazar, and I. Pop, "Stagnation flow of a micropolar fluid towards a vertical permeable surface," International Communications in Heat and Mass Transfer, vol. 35, no. 3, pp. 276281, 2008.

[9] Z. Ziabakhsh, G. Domairry, and H. Bararnia, "Analytical solution of non-Newtonian micropolar fluid flow with uniform suction/blowing and heat generation," Journal of the Taiwan Institute of Chemical Engineers, vol. 40, no. 4, pp. 443-451, 2009.

[10] H. A. Attia, "Investigation of non-Newtonian micropolar fluid flow with uniform suction/blowing and heat generation," Turkish Journal of Engineering and Environmental Sciences, vol. 30, no. 6, pp. 359-365, 2006.

[11] T. Hayat, T. Javed, and Z. Abbas, "MHD flow of a micropolar fluid near a stagnation-point towards a non-linear stretching surface," Nonlinear Analysis: Real World Applications, vol. 10, no. 3, pp. 1514-1526, 2009.

[12] T. Hayat, T. Javed, and Z. Abbas, "MHD flow of a micropolar fluid near a stagnation-point towards a non-linear stretching surface," Nonlinear Analysis: Real World Applications, vol. 11, no. 3, p. 2190, 2010.

[13] N. A. Yacob, A. Ishak, and I. Pop, "Melting heat transfer in boundary layer stagnation-point flow towards a stretching/shrinking sheet in a micropolar fluid," Computers \& Fluids, vol. 47, pp. 16-21, 2011.

[14] K. Das, "Slip effects on MHD mixed convection stagnation point flow of a micropolar fluid towards a shrinking vertical sheet," Computers \& Mathematics with Applications, vol. 63, no. 1, pp. 255-267, 2012.

[15] L. Zheng, J. Niu, X. Zhang, and L. Ma, "Dual solutions for flow and radiative heat transfer of a micropolar fluid over stretching/shrinking sheet," International Journal of Heat and Mass Transfer, vol. 55, pp. 7577-7586, 2012.

[16] A. Ishak, Y. Y. Lok, and I. Pop, "Stagnation-point flow over a shrinking sheet in a micropolar fluid," Chemical Engineering Communications, vol. 197, no. 11, pp. 1417-1427, 2010.

[17] G. Ahmadi, "Self-similar solution of imcompressible micropolar boundary layer flow over a semi-infinite plate," International Journal of Engineering Science, vol. 14, no. 7, pp. 639-646, 1976.

[18] A. Yücel, "Mixed convection in micropolar fluid flow over a horizontal plate with surface mass transfer," International Journal of Engineering Science, vol. 27, no. 12, pp. 1593-1602, 1989.

[19] Y. Jaluria and K. E. Torrance, Computational Heat Transfer, Taylor and Francis, New York, NY, USA, 2003.

[20] W. M. K. A. Wan Zaimi and A. Ishak, "Stagnation flow of a micropolar fluid towards a vertical permeable surface with prescribed heat flux," Sains Malaysiana, vol. 41, pp. 1263-1270, 2012.

[21] J. H. Merkin, "On dual solutions occurring in mixed convection in a porous medium," Journal of Engineering Mathematics, vol. 20, no. 2, pp. 171-179, 1985.

[22] P. D. Weidman, D. G. Kubitschek, and A. M. J. Davis, “The effect of transpiration on self-similar boundary layer flow over moving surfaces," International Journal of Engineering Science, vol. 44, no. 11-12, pp. 730-737, 2006.

[23] J. Paullet and P. Weidman, "Analysis of stagnation point flow toward a stretching sheet," International Journal of Non-Linear Mechanics, vol. 42, no. 9, pp. 1084-1091, 2007.
[24] S. D. Harris, D. B. Ingham, and I. Pop, "Mixed convection boundary-layer flow near the stagnation point on a vertical surface in a porous medium: Brinkman model with slip," Transport in Porous Media, vol. 77, no. 2, pp. 267-285, 2009.

[25] A. Postelnicu and I. Pop, "Falkner-Skan boundary layer flow of a power-law fluid past a stretching wedge," Applied Mathematics and Computation, vol. 217, no. 9, pp. 4359-4368, 2011.

[26] A. V. Rosca and I. Pop, "Flow and heat transfer over a vertical permeable stretching/shrinking sheet with a second order slip," International Journal of Heat and Mass Transfer, vol. 60, pp. 355364, 2013. 


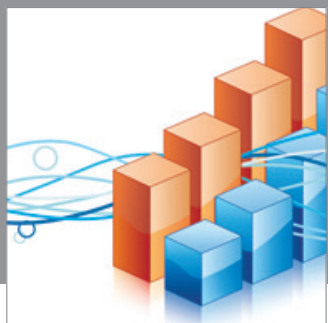

Advances in

Operations Research

mansans

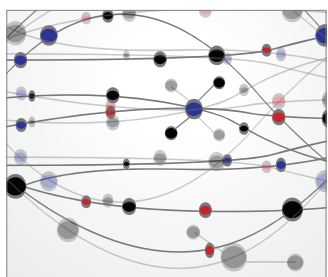

The Scientific World Journal
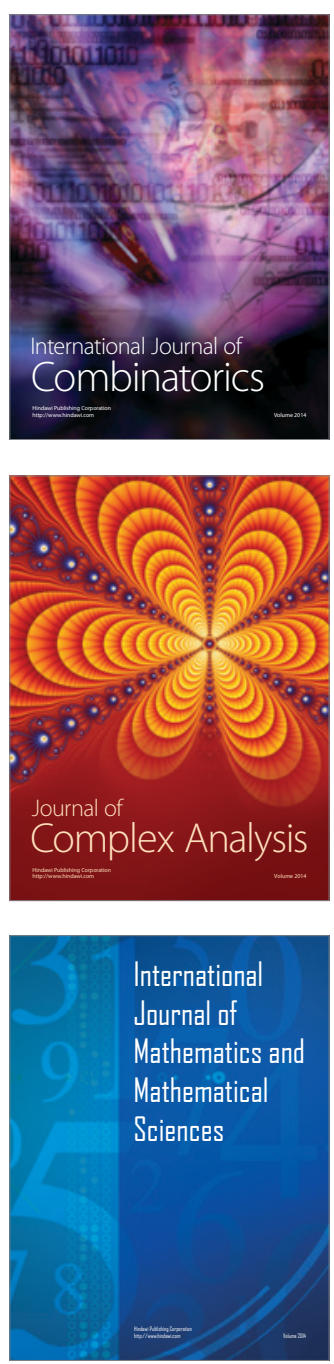
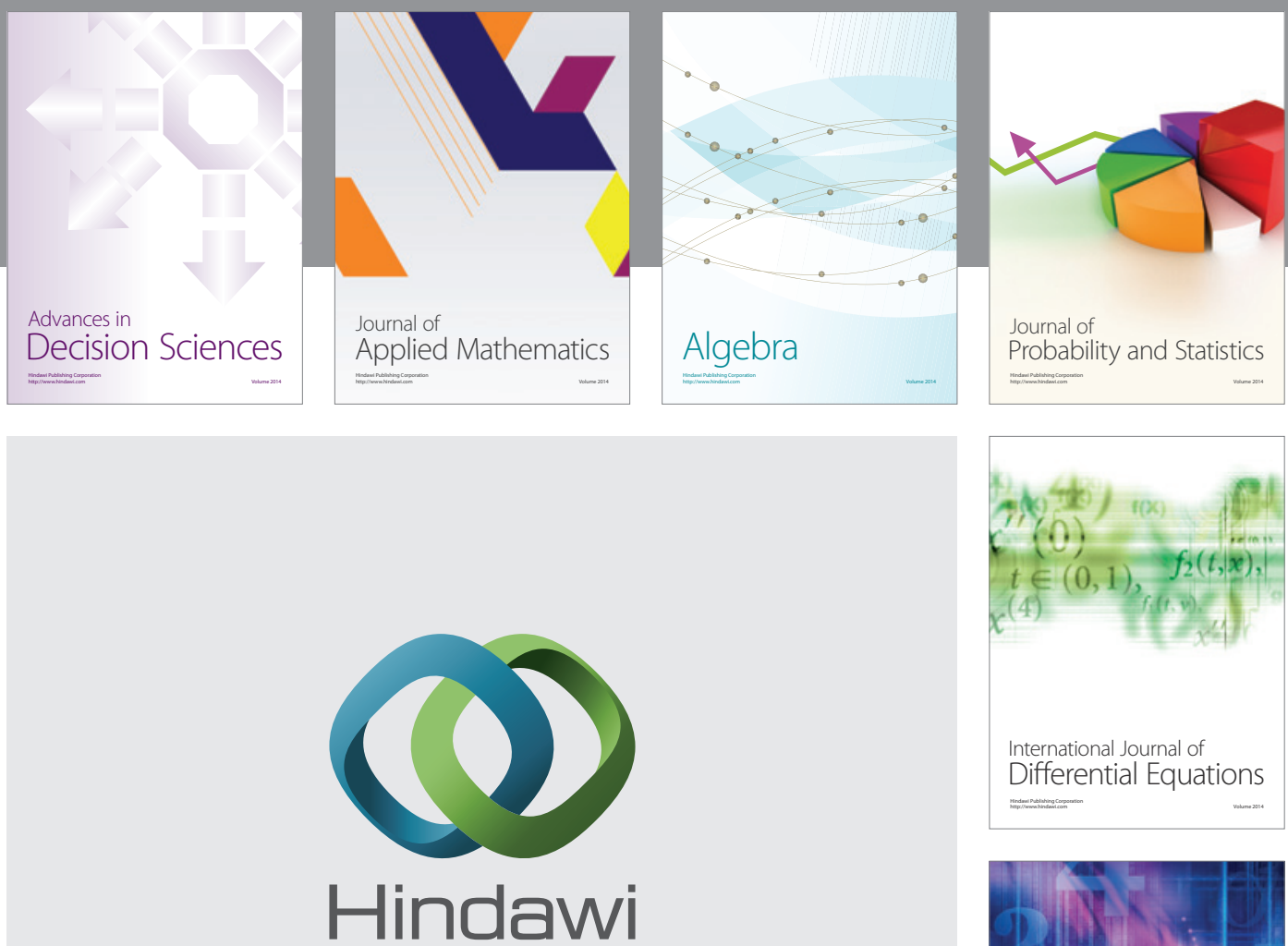

Submit your manuscripts at http://www.hindawi.com
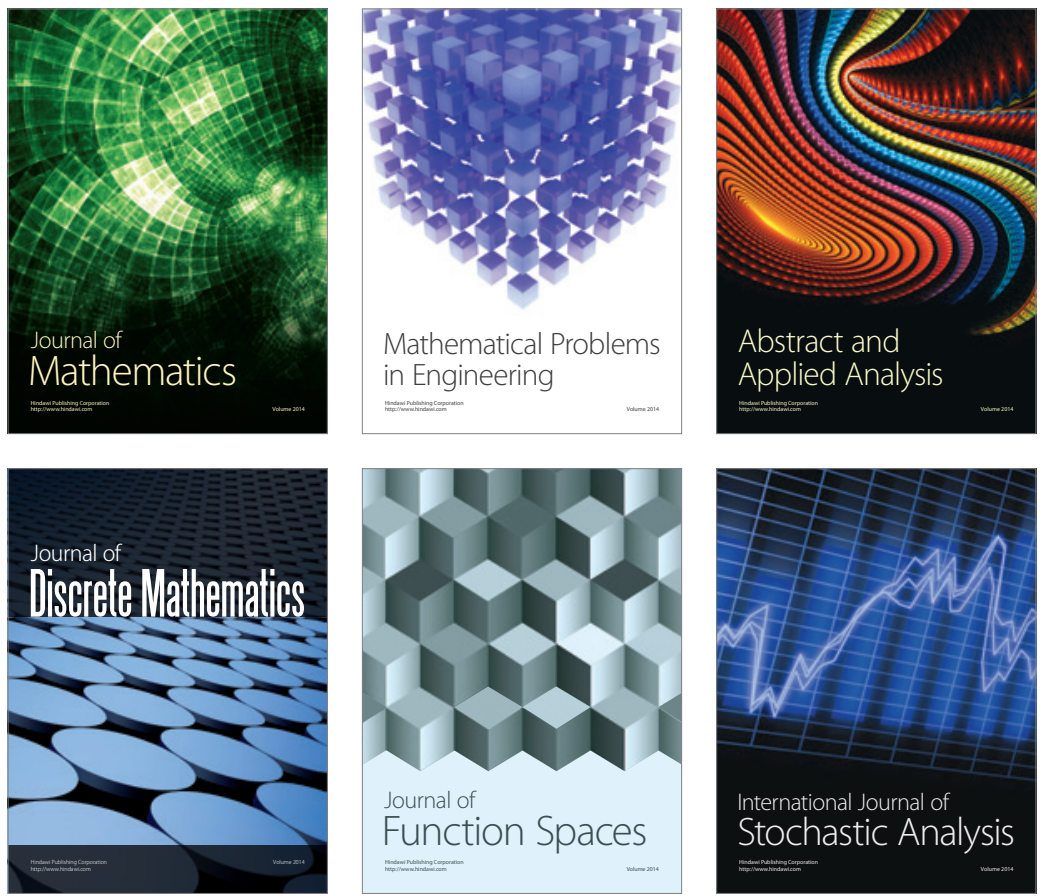

Journal of

Function Spaces

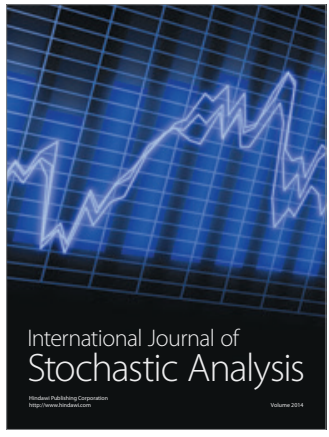

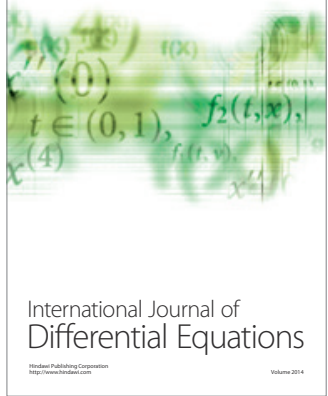
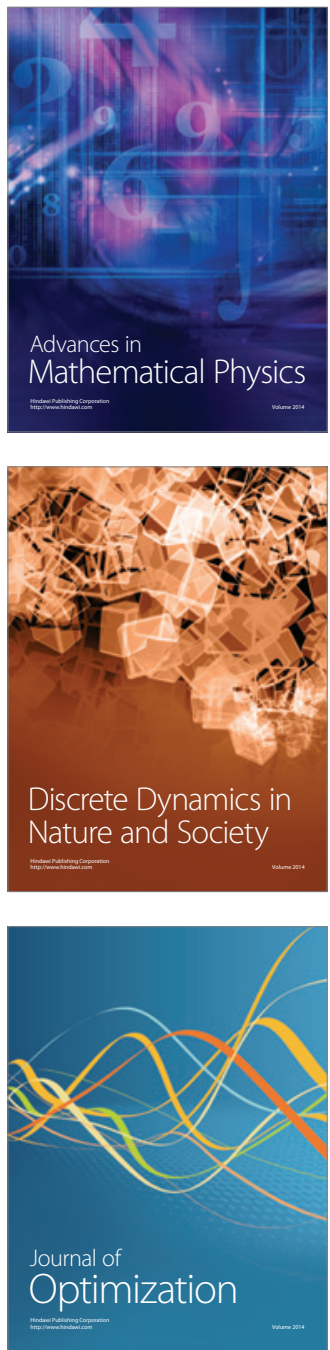